\title{
Ein Metallflachbeil der Kupferzeit aus Bernstein, Burgenland
}

\author{
Roland Haubner ${ }^{1}$, Susanne Strobl' ${ }^{1}$, Manuela Thurner ${ }^{2}$ und Hannes Herdits ${ }^{3}$ \\ ${ }^{1}$ Technische Universität Wien, Wien, Österreich \\ 2Institut für Urgeschichte und Historische Archäologie, Universität Wien, Wien, Österreich \\ ${ }^{3}$ Burgenländisches Landesmuseum, Eisenstadt, Österreich
}

Eingegangen 12. März 2020; angenommen 19. März 2020; online publiziert 16. April 2020

Zusammenfassung: Ein Kupferflachbeil des 4. Jahrtausends v.Chr., welches der Kulturstufe Balaton-Lasinja zuzuordnen ist, wurde bei Bernstein im Südburgenland gefunden und untersucht.

Die RFA Analyse ergab nahezu reines Kupfer mit geringen Mengen an $\mathrm{Pb}, \mathrm{Ag}$, As und Al. Metallographisch präparierte Schliffe zeigen ein sehr gleichmäßiges Gefüge mit feinem $\mathrm{Cu}-\mathrm{Cu}_{2} \mathrm{O}$ Eutektikum. Der O Gehalt im Metall wird auf etwa $0,2 \mathrm{Gew}$. \% geschätzt. Die Oberfläche des Beils ist mit einer etwa $100 \mu \mathrm{m}$ dicken Oxidschicht gleichmäßig bedeckt, welche innen aus $\mathrm{Cu}_{2} \mathrm{O}$ und außen aus Malachit besteht. Die Größe der einzelnen Kupferkörner ist mit 300-400 $\mu$ m eher grob, wobei auch einige Zwillingskristalle vorliegen. Daher ist anzunehmen, dass nur eine schwache Verformung des Beils, z. B. durch Hämmern, erfolgte.

Aufgrund der geringen Konzentrationen an $\mathrm{S}$ und As kann davon ausgegangen werden, dass oxydische Erze für die Kupfergewinnung verwendet wurden.

Schlüsselwörter: Kupferbeil, Mondsee-Kultur (Kulturstufe Balaton-Lasinja), $\mathrm{Cu}-\mathrm{Cu}_{2} \mathrm{O}$ Eutektikum

\section{A Copper Axe of the Mondsee Culture (Balaton-Lasinja)} Found in Bernstein, Burgenland

Abstract: A copper flat axe of the 4th millennium BC, which can be assigned to the Mondsee culture (middle Copper Age-Balaton-Lasinja phase) was found at Bernstein in southern Burgenland.

The XRF analysis revealed almost pure copper with small amounts of $\mathrm{Pb}, \mathrm{Ag}, \mathrm{As}$, and $\mathrm{Al}$. Metallographically prepared cross sections show a very uniform structure with fine $\mathrm{Cu}-\mathrm{Cu}_{2} \mathrm{O}$ eutectic. The $\mathrm{O}$ content in the metal is estimated to be about $0.2 \%$ by weight. The surface of the axe is evenly covered with an approximately $100 \mu \mathrm{m}$ thick oxide

Prof. Dr. R. Haubner ( $\bowtie)$

Technische Universität Wien,

Getreidemarkt 9/164-03,

1060 Wien, Österreich

roland.haubner@tuwien.ac.at layer, which consists of $\mathrm{Cu}_{2} \mathrm{O}$ on the inside and malachite on the outside. The size of the individual copper grains is rather coarse with $300-400 \mu \mathrm{m}$, although some twin crystals are present. It can be assumed that only a slight deformation of the axe occurred, e.g. by hammering.

Due to the low concentrations of $\mathrm{S}$ and As, it is likely that oxide ores were used for copper smelting.

Keywords: Copper axe, Mondsee culture (BalatonLasinja), $\mathrm{Cu}-\mathrm{Cu}_{2} \mathrm{O}$ eutectic

\section{Ein „Mondseeflachbeil” aus Bernstein, Burgenland}

Das hier beschriebene archäologische Fundobjekt ist ein in zwei Teile zerbrochenes Kupferflachbeil des 4. Jahrtausends v. Chr. Dieses wurde Anfang der 2000er Jahre im Gemeindegebiet von Bernstein im Südburgenland gefunden (BLM Inv. Nr. A-154197) (Abb. 1). Der Fundort ist eine markante, ursprünglich wahrscheinlich mit einem Abschnittswall befestigte Rückfallkuppe in $660 \mathrm{~m}$ Seehöhe.

Typologisch gleicht es den Beilen, die zahlreich im Bereich der sogenannten Mondseegruppe gefunden wurden, im zeitgleichen Balaton-Lasinja-Kreis des Burgenlandes aber außerordentlich selten sind [1]. Zwei weitere Beile dieses Typs, eines aus Miedlingsdorf (Inv.Nr. BLM A-18117) das zweite aus Gols (Inv.Nr. BLM A-29749), stammen aus Fundumständen, die eher an bäuerliche Siedlungen dieses Zeithorizontes denken lassen. In unmittelbarer Nähe des Fundortes in Bernstein gibt es solche Siedlungen jedoch nicht.

Die sogenannte "Mondseekultur" ist nur schwer von den benachbarten kupferzeitlichen Kulturkreisen abzugrenzen [2]. Aus dem Zeitraum von etwa 3800 bis 2900 v. Chr. wurden im Oberösterreichischen Alpenvorland zahlreiche Funde von Metallgusslöffeln und typischen Artefakten wie Flachbeilen, Dolchklingen und Spiraldrahtschmuck bekannt. Die klassische Mondseekeramik mit Tiefstichverzierung und Inkrustation ist das Leitfossil dieser Gruppe von metallverarbeitenden Sozietäten. Aufgrund der Über- 
schneidungen im Fundgut mit fast allen umgebenden Kulturkreisen müsste man die Gruppe eigentlich auf das Vorkommen der Mondseekeramik beschränken, das ist dann nur der unmittelbare Bereich des südlichen Oberösterreichs, wäre da nicht die sehr weite Verbreitung der am namengebenden Fundort so häufig gefundenen typischen Metallartefakte. Die Flachbeile der Mondseekultur erscheinen in einheitlicher Form in einem weiten Gebiet von Süddeutschland bis in das Karpatenbecken, wobei es unwahrscheinlich ist, dass alle in Oberösterreich produziert wurden.

Weil im Ostalpenraum viele kleine Kupfererzlagerstätten bekannt sind, herrschte lange Zeit die Meinung vor, die Metallfunde des beschriebenen Zeithorizontes wären aus den lokalen Erzen hergestellt worden. Neuere chemische und $\mathrm{Pb}$-Isotopenuntersuchungen weisen allerdings nach Südosteuropa [3]. Da dieses Beil typologisch eindeutig zur Mondseegruppe gehört, stellt sich die Frage nach dem Herstellungsort. Wurden lokale Erze verwendet, um vor Ort das Beil herzustellen oder wurde das Beil durch Handel bezogen [4]? Da es direkt am Fundort und im weiteren Bereich des sogenannten Bernstein-Rechnitzer Fensters zahlreiche Vorkommen von Kupferkies und den

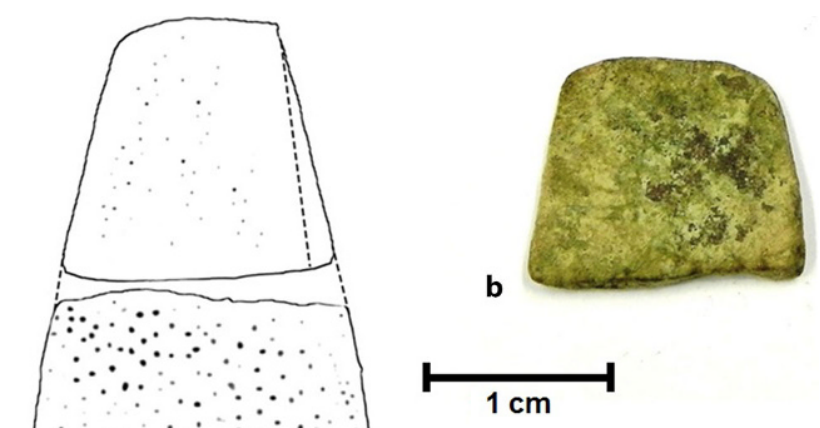

Abb. 1: Beil der Mondseekultur aus Bernstein/Kienberg. a Umzeichnung der beiden Bruchstücke (das Nackenbruchstück wurde dem Burgenländischen Landesmuseum überlassene; Inv.Nr. A-154197) Zeichnung: Manuela Thurner BA. b, c Vorder- und Rückseite des Nackenbruchstücks vor dem Abtrennen der Probe zur metallographischen Untersuchung
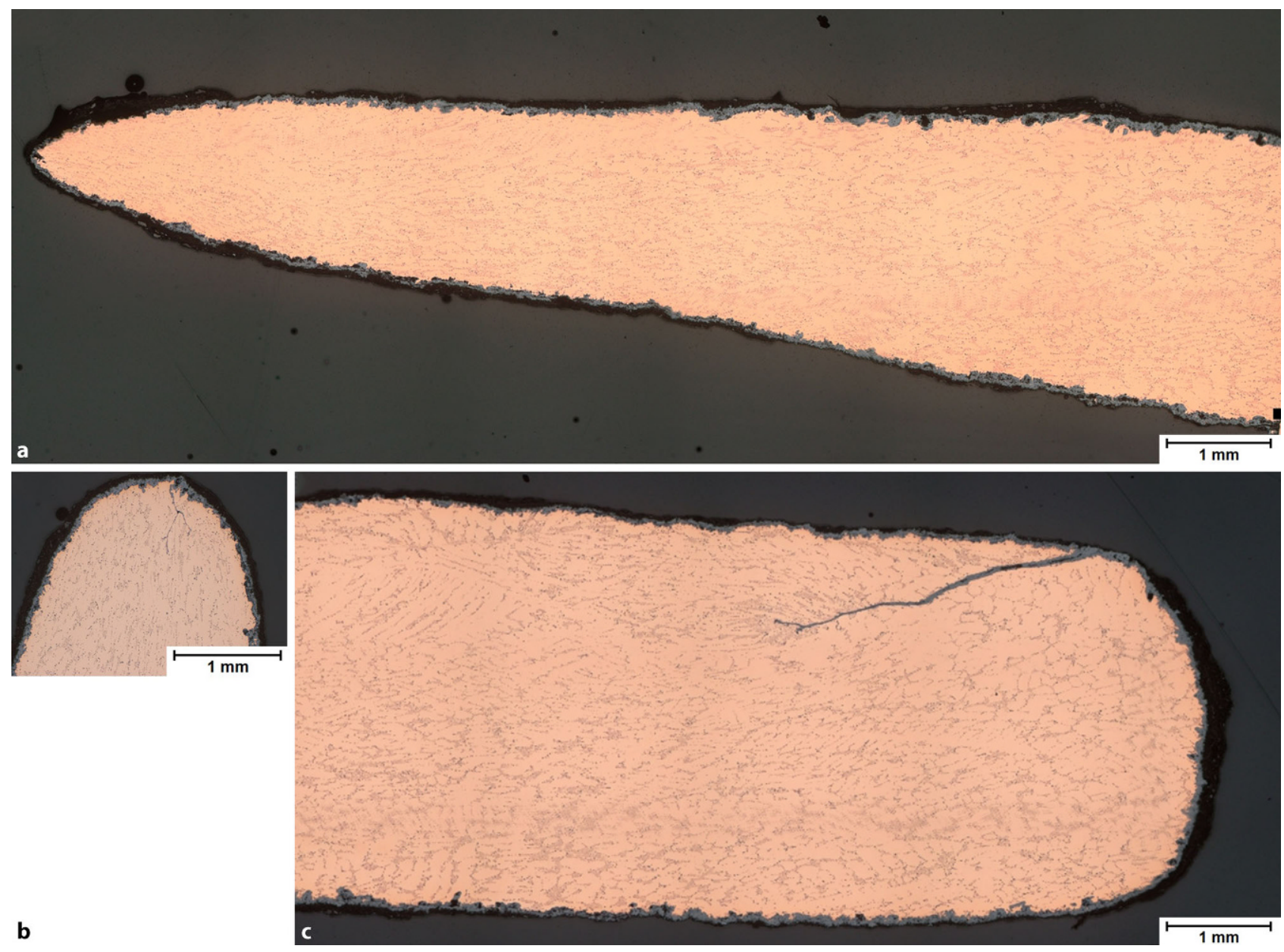

Abb. 2: Metallographische Schliffe des Kupferbeils: a Längsschnitt 1 mit Schneide; b Querschnitt; $\mathbf{c}$ Längsschnitt 2 mit Nackengrad 
entsprechenden sekundären Kupfermineralen gibt, wäre eine lokale Produktion denkbar.

\section{Das Beil}

Das Beil ist ohne Ansatz einer Biegung des Metalls gebrochen oder absichtlich zerschlagen worden. Das Kupferflachbeil mit einer Gesamtlänge von $7,5 \mathrm{~cm}$ und einer maximalen Breite von $4,2 \mathrm{~cm}$ wurde in zwei Teilen aufgefunden. Der kleinere Nackenteil mit einer Länge von $2,9 \mathrm{~cm}$ und einer Breite von 2,1 bzw. 1,8 cm weist am Bruch eine Stärke von bis zu $4 \mathrm{~mm}$ auf. Der Nackengrat selbst ist scharfkantig ausgebildet. Der bis zu $5 \mathrm{~mm}$ dicke Schneidenteil des Beiles weist eine Breite von 3,1 bis $4,2 \mathrm{~cm}$ auf. Die Länge des Schneidenbruchstücks beläuft sich auf $4,7 \mathrm{~cm}$. Die Schneide selbst ist, offenbar durch wiederholtes Nachschärfen, etwas abgeschrägt (Abb. 1).

\section{Metallkundliche Untersuchungen}

Das kleinere Bruchstück des Beils konnte metallographisch untersucht werden. Es wurden die Methoden 3D-Digitalmikroskop (3D-DM), Röntgenfluoreszenzanalyse (RFA), Metallographie, Lichtmikroskop (LOM) und Rasterelektronenmikroskop (REM) mit Energiedispersiver Röntgenanalyse (EDX) eingesetzt.

Zwei Längsschnitte (Abb. 2a, c) sowie ein Querschnitt (Abb. 2b) zeigen ein sehr gleichmäßiges Gefüge mit feinen Ausscheidungen im Inneren und einer Korrosionsschicht außen. An der Außenseite des Querschnitts sind ein schmaler etwa 0,5 mm langer Riss (Abb. 2b) und am dickeren En-

\begin{tabular}{|c|c|c|}
\hline \multicolumn{3}{|c|}{$\begin{array}{l}\text { TABELLE } 1 \\
\text { Chemische Zusammensetzung des Beils bestimmt } \\
\text { mittels RFA }\end{array}$} \\
\hline Gew.\% & Oberfläche & Schnittfläche \\
\hline $\mathrm{Cu}$ & 97,87 & 99,64 \\
\hline As & n.n. & 0,19 \\
\hline $\mathrm{Pb}$ & 1,69 & n.n. \\
\hline $\mathrm{S}$ & 0,05 & 0,03 \\
\hline $\mathrm{Al}$ & 0,21 & 0,01 \\
\hline $\mathrm{Si}$ & 0,07 & 0,05 \\
\hline $\mathrm{P}$ & 0,04 & 0,04 \\
\hline $\mathrm{Ag}$ & 0,02 & n.n. \\
\hline $\mathrm{Fe}$ & 0,03 & 0,03 \\
\hline Co & n.n. & n.n. \\
\hline $\mathrm{Ni}$ & 0,01 & 0,01 \\
\hline $\mathrm{Sb}$ & n.n. & n.n. \\
\hline Sn & n.n. & n.n. \\
\hline $\mathrm{Bi}$ & n.n. & n.n. \\
\hline $\mathrm{Zn}$ & n.n. & n.n. \\
\hline $\mathrm{Mn}$ & n.n. & n.n. \\
\hline $\mathrm{Cr}$ & n.n. & n.n. \\
\hline $\mathrm{V}$ & n.n. & n.n. \\
\hline $\mathrm{Ti}$ & 0,01 & n.n. \\
\hline
\end{tabular}

de des Längsschnitts ein etwa $8 \mathrm{~mm}$ langer, etwas breiterer Riss (Abb. 2c) zu sehen. Beide Risse enthalten Oxidationsprodukte von Kupfer.

Eine RFA Analyse der Beiloberfläche ergab nahezu reines Kupfer mit geringen Mengen an $\mathrm{Pb}$ und $\mathrm{Al}$ (Tab. 1).
Abb. 3: a Korrodierte Beiloberfläche im 3D-DM; b Schneide des Beils im LOM; c Korrosionsschicht im LOM; d Korrosionsschicht im LOM mit polarisiertem Licht
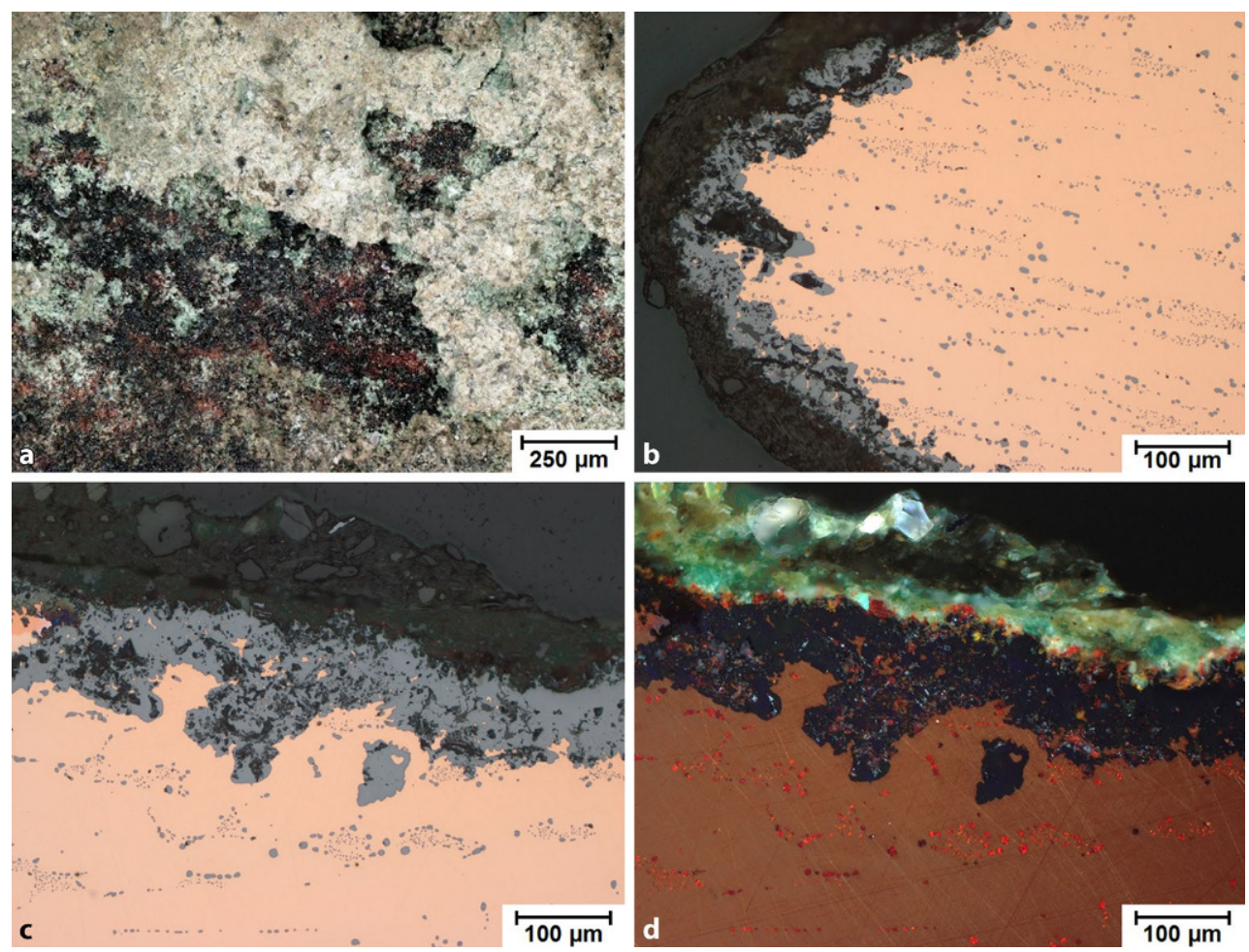
Abb. 4: $\mathrm{Cu}-\mathrm{Cu}_{2} \mathrm{O}$ Eutektikum im Gefüge des Beils: a Cu-Cu2 $\mathrm{O}$ Eutektikum im LOM; $\mathbf{b} \mathrm{Cu}-\mathrm{Cu}_{2} \mathrm{O}$ Eutektikum im LOM mit polarisiertem Licht; $\mathrm{c} C \mathrm{Cu}-\mathrm{Cu}_{2} \mathrm{OEu}$ tektikum im REM; d Sauerstoffverteilung gemessen mittels REM-EDX
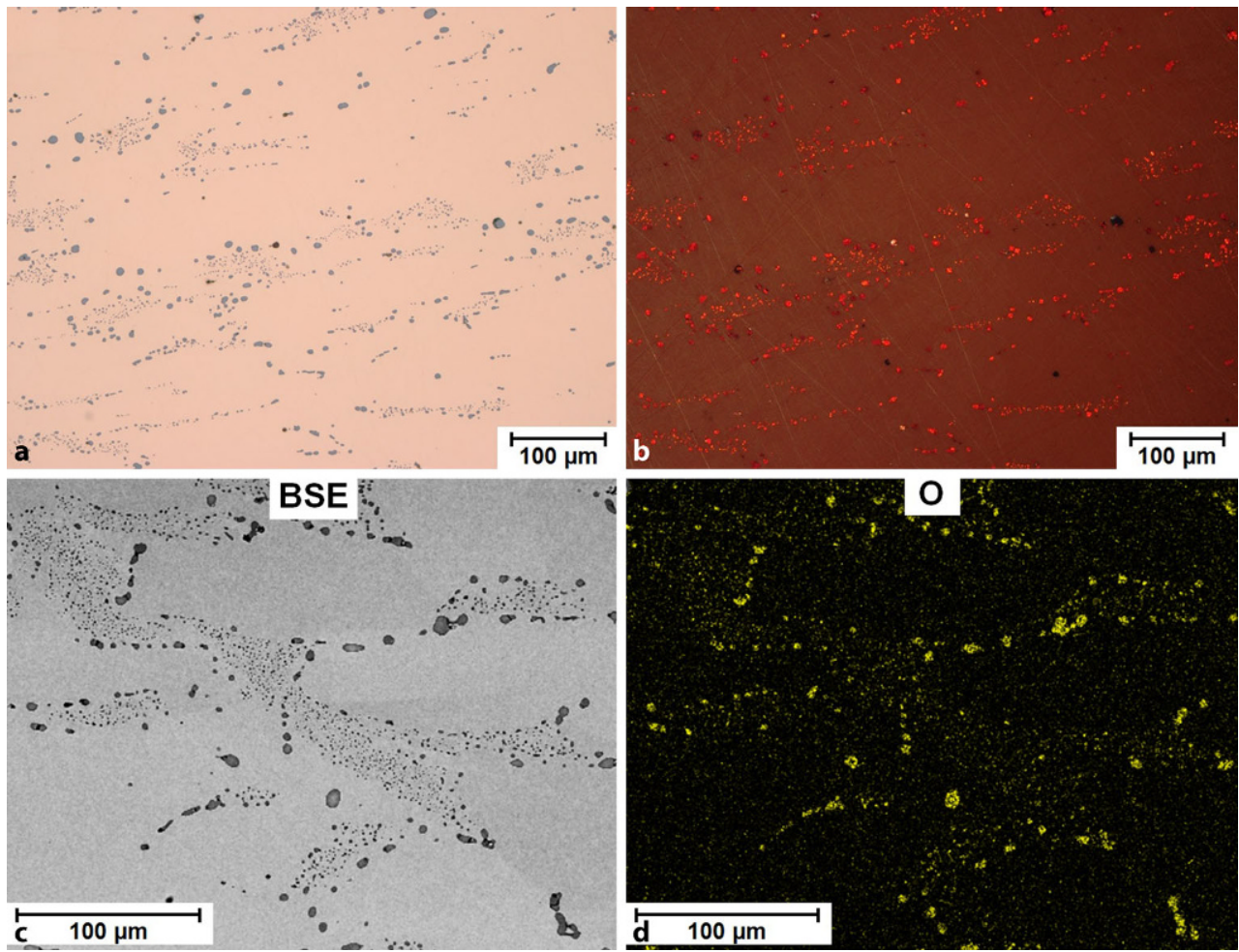

Die Konzentrationen weiterer Spurenelemente waren unter 0,1 Gew.\%. Der Vergleich mit einer RFA Analyse an einer Schnittfläche zeigt geringe Unterschiede zwischen dem metallischen Kern und der Patina auf. So wurden in der Patina höhere Konzentrationen an $\mathrm{Pb}, \mathrm{Ag}$ und $\mathrm{Al}$ gemessen. Dies könnte einerseits auf eine Anreicherung während der Korrosion (Patinabildung) oder andererseits auf einen Eintrag aus der Umgebung zurückzuführen sein [5].

Die Oberfläche des Beils ist mit einer etwa $100 \mu \mathrm{m}$ dicken Oxidschicht gleichmäßig bedeckt (Abb. 3a,b), welche zweischichtig aufgebaut ist (Abb. 3c,d). Der Aufbau der Oxidschicht ist deutlich im polarisierten Licht zu erkennen, da der außenliegende Malachit grün und darunterliegendes $\mathrm{Cu}_{2} \mathrm{O}$ dunkelrot bis schwarz erscheint (Abb. 3d). Eine 3D-DM Abbildung der Beilklingenoberfläche ist in Abb. 3a zu sehen. Die hellgrünen Bereiche entsprechen dem Mineral Malachit und das darunterliegende dunkle Material ist $\mathrm{Cu}_{2} \mathrm{O}$.

Das metallische Grundmetall ist eine Mischung aus Kupfer und $\mathrm{Cu}-\mathrm{Cu}_{2} \mathrm{O}$ Eutektikum, das bei der Erstarrung der Schmelze entstanden ist (Abb. 4a). Das $\mathrm{Cu}_{-} \mathrm{Cu}_{2} \mathrm{O}$ Eutektikum liegt bei einem Sauerstoffgehalt von 0,4 Gew. \% im Cu [6]. Da nicht das gesamte Gefüge aus Eutektikum besteht, sondern nur zu etwa $50 \%$, kann der O Gehalt im Metall auf etwa 0,2 Gew. \% geschätzt werden. Im polarisierten Licht ist das $\mathrm{Cu}_{2} \mathrm{O}$ an der hellroten Farbe gut deutlich erkennbar (Abb. 4b; [7]). In den REM Bildern ist $\mathrm{Cu}_{2} \mathrm{O}$ dunkler als das metallische $\mathrm{Cu}$ (Abb. 4c). Um die Anwesenheit von $\mathrm{Cu}_{2} \mathrm{O}$ zu bestätigen, wurde mittels REM-EDX eine Elementverteilung für $\mathrm{O}$ gemessen (Abb. 4d). In der Literatur wurde ein ähnliches Gefüge wie hier auch beim sogenannten „ÖtziBeil" beschrieben $[8,9]$.
Durch Ätzungen kann das Gefüge des Kupfers sichtbar gemacht werden (Abb. 5a). Die Größe der einzelnen Kupferkörner ist nur schwer zu bestimmen, denn vorhandene Zwillingskristalle und Farbunterschiede verhindern dies (Abb. 5b). Die Korngröße wird auf 300-400 $\mu$ m geschätzt, was relativ grob ist. Die Farbunterschiede nach der Ätzung können durch minimale Unterschiede in den Spurenelementen hervorgerufen werden. Da die Anzahl der Zwillingskristalle eher gering ist (Abb. $5 c, d$ ), kann davon ausgegangen werden, dass nur schwache Verformung des Beils, z. B. durch Hämmern, erfolgte.

\section{Das Metallurgieverständnis der frühen bis mittleren Kupferzeit}

Offenbar dürfte sich bei den metallurgischen Handwerksmeistern des Zeitraumes von etwa 5000 bis 3000 v. Chr. an der Werkanleitung „mach die bunten Steine im Feuer so heiß, bis das rote unzerbrechliche Material entsteht" nicht viel geändert haben. Auch eine mit Sicherheit beabsichtigte Legierung mit Arsen, Antimon oder anderen brauchbaren Verbesserern ist nicht eindeutig nachzuweisen, sondern dürfte durch die Lagerstätten bedingt sein. Erst die allgemeine Einführung der Zinnbronze lässt Stein als Werkstoff verschwinden, und der dann stetig steigende Metallbedarf führt zur Entwicklung industriell vollzogener Technologien und schließlich in der späten Bronzezeit zu modern anmutenden mehrstufigen Verhüttungsprozessen [10].

In der Kupferzeit bestand die Erzbasis für die Kupfergewinnung somit aus oxidischen Erzen, wobei der Malachit $\mathrm{Cu}_{2}(\mathrm{OH})_{2}\left(\mathrm{CO}_{3}\right)$ durch seine leuchtend grüne Farbe beson- 

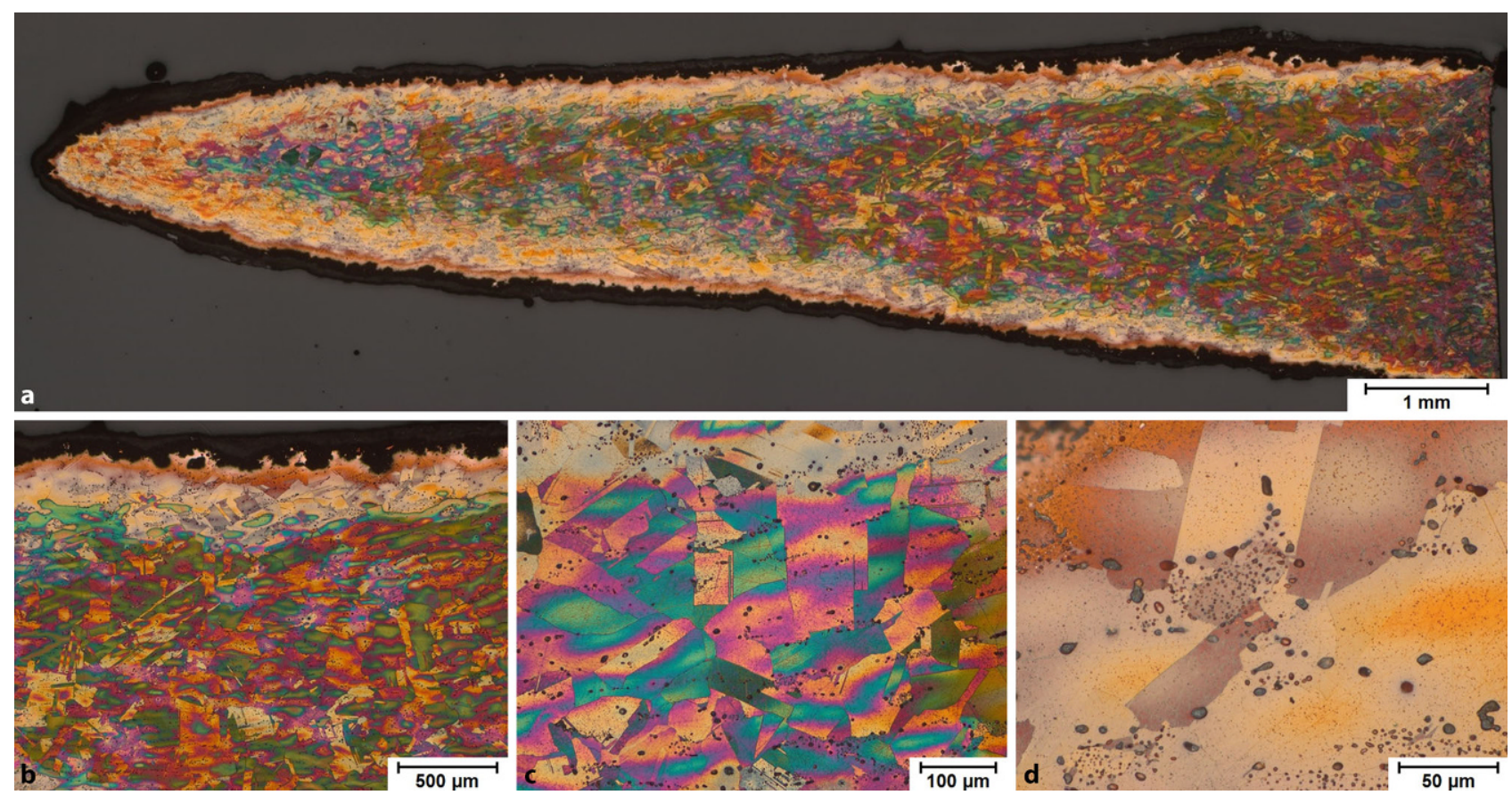

Abb. 5: Ätzung des Beils mit Klemm 2 Lösung (LOM): a Übersicht Schrägschliff 1; b Randbereich des Beils; c grobkörniges Kupfergefüge mit Zwillingskorngrenzen; d Detailaufnahme mit Cu-Cu $2 \mathrm{O}$ Eutektikum und Zwillingskorngrenzen

ders leicht aufzufinden war. Für die Herkunftsbestimmung des Kupfers ist, vor allem bei sehr reinem $\mathrm{Cu}$, eine chemische Analyse nicht zielführend. Ob eine Bestimmung der Blei-Isotopenverhältnisse ein brauchbares Ergebnis liefern würde, ist nicht vorherzusagen. Der Umstand, dass im vorliegenden Beil nur Spuren an S und As vorhanden sind, deutet somit auf ein oxydisches Erz als Ausgangsmaterial.

Die Verhüttung des oxidischen Erzes zu metallischen $\mathrm{Cu}$ ist einfach und kann in einem flachen Tiegel oder Herd mit Holzkohle als Reduktionsmittel, erfolgen. Von der Dauer des Reduktionsschritts war es nun abhängig, wieviel Sauerstoff noch im $\mathrm{Cu}$ gelöst war und wieviel Cuprit $\left(\mathrm{Cu}_{2} \mathrm{O}\right)$ bei der Erstarrung, beim sogenannten $\mathrm{Cu}-\mathrm{Cu}_{2} \mathrm{O}$ Eutektikum, gebildet wurde. Aus der Mondseekultur der späten Jungsteinzeit sind Gießlöffel bekannt, in denen das Cu mittels Blasrohren aufgeschmolzen wurde. Durch das Vorliegen eines Eutektikums konnte bereits beim Schmelzpunkt von $\mathrm{Cu}\left(1085^{\circ} \mathrm{C}\right)$ eine vollständig aufgeschmolzene Legierung erhalten werden.

Das Auftreten der ältesten Arsen-Bronzen wird üblicherweise mit der Verhüttung von Fahlerzen oder deren Sekundärprodukten erklärt [11].

Die Verhüttung von Fahlerz ist relativ einfach, denn das Erz muss nur möglichst vollständig geröstet werden, um ein Oxid zu erhalten. Beim Rösten verflüchtigen sich As und $S$ weitgehend [12]. Die anschließende Reduktion kann wieder mit Holzkohle erfolgen, wobei beim Rösten verbliebenes As im geschmolzenen $\mathrm{Cu}$ aufgenommen wird. Während weiterer Schmelzvorgänge und dem Gießvorgang, verbleibt das As in der Schmelze und verflüchtigt sich nicht [13].

\section{Zusammenfassung}

Ein im Gemeindegebiet von Bernstein im Südburgenland gefundenes Kupferflachbeil des 4. Jahrtausends v. Chr. wurde untersucht. Typologisch ist dieses Beil eindeutig der sogenannten Mondseegruppe (Zeitstufe Balaton-Lasinja) zuzuordnen, wodurch sich die Frage nach dem Herstellungsort stellt. Wurde das Beil vor Ort aus lokalen Erzen hergestellt oder durch Handel bezogen?

Die RFA Analyse ergab nahezu reines Kupfer mit geringen Mengen an $\mathrm{Pb}, \mathrm{Ag}$, As und Al. Metallographisch präparierte Schnitte zeigen ein sehr gleichmäßiges Gefüge mit feinen $\mathrm{Cu}_{2} \mathrm{O}$ Ausscheidungen im Inneren, sowie einer außenliegenden Korrosionsschicht. Außerdem wurden zwei Risse, gefüllt mit Oxidationsprodukten von Kupfer, gefunden. Die Oberfläche des Beils ist mit einer etwa $100 \mu \mathrm{m}$ dicken Oxidschicht gleichmäßig bedeckt, welche innen aus $\mathrm{Cu}_{2} \mathrm{O}$ und außen aus Malachit besteht. Das metallische Grundmetall ist eine Mischung aus Kupfer und $\mathrm{Cu}-\mathrm{Cu}_{2} \mathrm{O}$ Eutektikum, wobei der O Gehalt im Metall auf etwa 0,2 Gew. \% geschätzt wird. Mittels REM-EDX Elementverteilung wurde bestätigt, dass ein $\mathrm{Cu}-\mathrm{Cu}_{2} \mathrm{O}$ Eutektikum vorliegt.

Die Größe der einzelnen Kupferkörner ist relativ grob und wird auf 300-400 $\mu \mathrm{m}$ geschätzt, wobei auch einige Zwillingskristalle vorliegen. Es kann davon ausgegangen werden, dass nur schwache Verformung des Beils, z. B. durch Hämmern, erfolgte.

$\mathrm{Da}$ das Beil weder höhere Konzentrationen an S noch an As enthält, kann davon ausgegangen werden, dass oxydische Erze für die Kupfergewinnung verwendet wurden. Woher diese Erze stammen könnte vielleicht eine $\mathrm{Pb}$ Isotopenbestimmung klären. 
Funding. Open access funding provided by TU Wien (TUW).

Open Access Dieser Artikel wird unter der Creative Commons Namensnennung 4.0 International Lizenz veröffentlicht, welche die Nutzung, Vervielfältigung, Bearbeitung, Verbreitung und Wiedergabe in jeglichem Medium und Format erlaubt, sofern Sie den/die ursprünglichen Autor(en) und die Quelle ordnungsgemäß nennen, einen Link zur Creative Commons Lizenz beifügen und angeben, ob Änderungen vorgenommen wurden.

Die in diesem Artikel enthaltenen Bilder und sonstiges Drittmaterial unterliegen ebenfalls der genannten Creative Commons Lizenz, sofern sich aus der Abbildungslegende nichts anderes ergibt. Sofern das betreffende Material nicht unter der genannten Creative Commons Lizenz steht und die betreffende Handlung nicht nach gesetzlichen Vorschriften erlaubt ist, ist für die oben aufgeführten Weiterverwendungen des Materials die Einwilligung des jeweiligen Rechteinhabers einzuholen.

Weitere Details zur Lizenz entnehmen Sie bitte der Lizenzinformation auf http://creativecommons.org/licenses/by/4.0/deed.de.

\section{Literatur}

1. Obereder, J.; Ruttkay, J.; Pernicka, E.: Die Metallfunde und die Metallurgie der kupfer-zeitlichen Mondseegruppe. Ein Vorbericht, Archäologie Österreichs, 4 (1993), Nr. 2, S. 5-9

2. Ruttkay, E.; Cichocki, O.; Pernicka, E.; Pucher, E.: Prehistoric lacustrine villages on the Austrian lakes: past and recent research developments. in: Menotti, F. (ed.): Living on the lake in prehistoric Europe. Routledge, 2004, S. 50-68

3. Pernicka, E.; Frank, C.: Das Kupfer der Mondseegruppe. in: Stöllner, T., Oeggl, K. (Hrsg.): Bergauf, Bergab, 10000 Jahre Bergbau in den Ostalpen. Veröffentlichungen aus dem Deutschen Bergbaumuseum Bochum, 207 (2015), S. 77-82
4. Artioli, G.; Angelini, I.; Kaufmann, G.; Canovaro, C.; Dal Sasso, G.; Villa I. M.: Long-distance connections in the Copper Age: new evidence from the Alpine Iceman's copper axe, PLoS ONE 2017, https:// doi.org/10.1371/journal.pone.0179263

5. Chang, T.; Wallinder, I. O.; de la Fuente, D.; Chico, B.; Morcillo, M.; Welter, J.-M.; Leygraf, C.: Analysis of Historic Copper Patinas. Influence of Inclusions on Patina Uniformity, Materials, 298 (2017), no 10, pp 1-13

6. Massalski, T. B.: Binary Alloy Phase Diagrams, ASM International, Metals Park OH, 1990.

7. Undisz, A.; Wilke, J.; Kolbe, H.; Seeger, J.; Rettenmayr, M.: The Copper of the Kyffhäuser Monument, Pract. Metallogr., 54 (2017), pp 353-365

8. Sperl, G.: Der Werkstoff des "Ötzibeiles" Die kupferzeitliche Beilklinge der Gletschermumie aus den Ötztaler Alpen, BHM Berg- und Hüttenmännische Monatshefte, 163 (2018), S. 451-455

9. Spindler, K.: Das Beil vom Hauslabjoch mit seinem archäologischen Umfeld, in: Weisgerber, G.; Goldenberg, G. (Hrsg.): Alpenkupfer = Rame delle Alpi, Bochum: Dt. Bergbau-Museum, Der Anschnitt, Beiheft, 17 (2004), S. 228

10. Herdits, H.: Die ostalpine bronzezeitliche Kupfererzeugung im überregionalen Vergleich am Grundbeispiel eines Hüttenplatzes in Mühlbach/Sbg., Dissertation an der Universität Wien, Wien, 2017

11. Budd, P.: A metallographic investigation of eneolithic arsenical copper artefacts from mond-see, Austria. J. Hist. Metall. Soc., 25 (1991), pp 99-108

12. Haubner, R.; Strobl, S.: Consideration about the metallurgy of $\mathrm{Cu}$ As Bronzes, in: Monographies Instrumentum, submitted 2020

13. Mödlinger, M.; Oro Calderon, R.; Haubner, R.: Arsenic loss during metallurgical processing of arsenical bronze, Archaeol Anthropol Sci, 11 (2019), pp 133-140

Hinweis des Verlags. Der Verlag bleibt in Hinblick auf geografische Zuordnungen und Gebietsbezeichnungen in veröffentlichten Karten und Institutsadressen neutral. 